\title{
On the Oscillatory and Asymptotic Behavior of the Bounded Solutions of Differential Equations with Deviating Arguments (*) (**).
}

\author{
Ch. G. PHilos (Ioannina, Greece)
}

Summary. - This paper is dealing with the oscillatory and asymptotic behavior of the bounded solutions of $n$-th order $(n>1)$ differential equations with deviating arguments involving the so called r-derivatives $D_{r}^{(i)} x(i=0,1, \ldots, n)$ of the unknown function $x$ defined by

$$
D_{r}^{(0)} x=x, \quad D_{r}^{(i)} x=r_{\imath}\left(D_{r}^{(i-1)} x\right)^{r} \quad(i=1,2, \ldots, n-1) \quad \text { and } \quad D_{r}^{(n)} x=\left(D_{r}^{(n-1)} x\right)^{l},
$$

where $r_{i}(i=1,2, \ldots, n-1)$ are positive continuous functions on the interval $\left[t_{0}, \infty\right)$. The fundamental purpose is to find a necessary and sufficient condition in order to have at least one (bounded nonoscillatory) solution whose the limit at $\infty$ exists in $\boldsymbol{R}-\{0\}$.

\section{1. - Introduction.}

Let $r_{i}(i=0,1, \ldots, n)$ be positive continuous functions on the interval $\left[t_{0}, \infty\right)$. For a real-valued function $h$ on $[T, \infty), T \geqq t_{0}$, and any $\mu=0,1, \ldots, n$ the $\mu$-th $r$-derivative of $h$ is defined by the formula

$$
D_{r}^{(\mu)} h=r_{\mu}\left(r_{\mu-1}\left(\ldots\left(r_{1}\left(r_{0} h\right)^{\prime}\right)^{\prime} \ldots\right)^{\prime}\right)^{\prime} .
$$

Then we obviously have

$$
D_{r}^{(0)} h=r_{0} h \quad \text { and } \quad D_{r}^{(i)} h=r_{i}\left(D_{r}^{(i-1)} h\right)^{\prime} \quad(i=1,2, \ldots, n) .
$$

If $D_{r}^{(n)} h$ is defined on $[T, \infty)$, then the function $h$ is called $n$-iimes $r$-differentiable and, if in addition $D_{r}^{(n)} h$ is continuous, $h$ is called $n$-times continuously r-differentiable. We note that in the case where $r_{0}=r_{1}=\ldots=r_{n}=1$ the above notion of $r$-differentiability spesializes to the usual one.

Now, we consider the $n$-th order $(n>1)$ differential equation with deviating arguments of the form

$$
\left(D_{r}^{(n)} x\right)(t)+a(t) \Phi\left(x\left\langle g_{0}(t)\right\rangle,\left(D_{r}^{(1)} x\right)\left\langle g_{1}(t)\right\rangle, \ldots,\left(D_{r}^{(N)} x\right)\left\langle g_{N}(t)\right\rangle\right)=b(t)
$$

(*) Entrata in Redazione il 29 giugno 1977.

(**) This paper is a part of the author's Doctoral Thesis submitted to the School of Physics and Mathematics of the University of Ioannina. 
where $r_{0}=r_{n}=1, N$ is an integer with $0 \leqq N \leqq n-1$ and

$$
\begin{aligned}
&\left(D_{r}^{(i)} x\right)\left\langle g_{i}(t)\right\rangle=\left(\left(D_{r}^{(i)} x\right)\left[g_{i 1}(t)\right],\left(D_{r}^{(i)} x\right)\left[g_{i 2}(t)\right], \ldots,\left(D_{r}^{(i)} x\right)\left[g_{i m_{i}}(t)\right]\right), \\
& g_{i}=\left(g_{i 1}, g_{i 1}, \ldots, g_{i m_{i}}\right) \quad(i=0,1, \ldots, N) .
\end{aligned}
$$

The continuity of the real-valued functions $a, g_{i j}\left(j=1,2, \ldots, m_{i} ; i=0,1, \ldots, N\right)$ and $b$ on $\left[t_{0}, \infty\right)$ and $\Phi$ on $\boldsymbol{R}^{m_{0}} \times \boldsymbol{R}^{m_{1}} \times \ldots \times \boldsymbol{R}^{m_{N}}$ as well as sufficient smoothness for the existence of solutions of $(E)$ on an infinite subinterval of $\left[t_{0}, \infty\right)$ will be assumed without mention. In what follows the term "solution" is always used only for such solutions $x(t)$ of $(E)$ which are defined for all large $t$. The oscillatory character is considered in the usual sence, i.e. a continuous real-valued funetion which is defined on an interval of the form $\left[T_{0}, \infty\right)$ is called oscillatory if it has no last zero, and otherwise it is called nonoseillatory.

Furthermore, the conditions (i) and (ii) below are assumed to hold throughout the paper:

(i) For $i=0,1, \ldots, N$ and every $j=1,2, \ldots, m_{i}$

$$
\lim _{t \rightarrow \infty} g_{i j}(t)=\infty \text {. }
$$

(ii) The function $\Phi$ has the sign property

$$
\left\{\begin{array}{l}
\left(\forall j=1,2, \ldots, m_{0}\right) y_{0 j}>0 \Rightarrow \Phi\left(y_{0}, y_{1}, \ldots, y_{N}\right)>0 \\
\left(\forall j=1,2, \ldots, m_{0}\right) y_{0 j}<0 \Rightarrow \Phi\left(y_{0}, y_{1}, \ldots, y_{N}\right)<0,
\end{array}\right.
$$

where $y_{i} \in \boldsymbol{R}^{m_{t}}(i=0,1, \ldots, N)$ and $y_{0}=\left(y_{01}, y_{02}, \ldots, y_{0 m_{0}}\right)$.

For such differential equations involving the operator $D_{r}^{(n)}$ there is recently an increasing interest in studying the oscillatory and asymptotic behavior of the solutions. We choose to refer here to the papers by Kusano and Orose [2, 3], PHILos [4, 5, 6], Philos and Staikos [7, 8], Stajkos and Philos [11, 12, 13] and Trench [14]. As one can see in the book of COPPEL [1], the class of operators $D_{r}^{(n)}$ properly contains the disconjugate operators $L$,

$$
L x=x^{(n)}+p_{1} x^{(n-1)}+\ldots+p_{n-1} x+p_{n} \quad\left(p_{i} \text { continuous functions }\right) .
$$

\section{2. - Main results.}

In order to obtain our first theorem (Theorem 1) we shall apply the fixed point technique, by using the following Schauder's theorem (see SCHAUDER [9]).

The SchaUder THEOREMr. - Let $E$ be $a$ Banach space and $X$ any nonempty convex and chosed subset of $E$. If $S$ is a continuous mapping of $X$ into itself and $S X$ is relatively compact, then the mapping $S$ has at least one fixed point (i.e. there exists an $x \in X$ with $x=S x)$. 
A set $\mathcal{F}$ of real-valued functions defined on the interval $[T, \infty)$ is said to be equiconvergent at $\infty$ if all functions in $\mathscr{F}$ are convergent in $\boldsymbol{R}$ at the point $\infty$ and, moreover, for every $\varepsilon>0$ there exists a $T^{\prime} \geqq T$ such that, for all $f \in \mathcal{F}$,

$$
t \geqq T^{\prime} \Rightarrow\left|f(t)-\lim _{\varepsilon \rightarrow \infty} f(s)\right|<\varepsilon
$$

Let now $B([T, \infty))$ be the Banach space of all continuous and bounded realvalued functions on the interval $[T, \infty)$, endowed with the usual sup-norm \|\| . We need the following compactness criterion for subsets of $B([T, \infty))$, which is a corollary of the Arzela-Ascoli theorem. For a proof of this etiterion we refer to STAIKos [10].

COMPACTNESS CRITERION. - Let $\mathscr{F}$ be an equicontinuous and uniformly bounded subset of the Banach space $B([T, \infty))$. If $\mathcal{F}$ is equiconvergent at $\infty$, it is also relatively compaet.

THEOREM 1. - Consider the differential equation (E) subject to the conditions (i), (ii) and:

$\left(O_{1}\right)$ There exists an $n$-times continuously $r$-differentiable function $w$ on $\left[t_{0}, \infty\right)$ with $D_{r}^{(n)} w=b$ and such that

$$
\begin{gathered}
A=\max _{0 \leqq i \leqq N} \limsup _{t \rightarrow \infty}\left|\left(D_{r}^{(i)} w\right)(t)\right|<\infty . \\
\int^{\infty} \frac{1}{r_{1}\left(s_{1}\right)} \int_{s_{1}}^{\infty} \frac{1}{r_{2}\left(s_{2}\right)} \ldots \int_{s_{n-2}}^{\infty} \frac{1}{r_{n-1}\left(s_{n-1}\right)} \int_{s_{n-1}}^{\infty}|a(s)| d s d s_{n-1} \ldots d s_{2} d s_{1}<\infty .
\end{gathered}
$$

Then for every real number $L$ with $|L|>2 A$ there exists a (bounded nonosoillatory) solution $x$ of the equation $(E)$ with

$$
\lim _{t \rightarrow \infty}[x(t)-w(t)]=L
$$

and

$$
\lim _{t \rightarrow \infty}\left[\left(D_{r}^{(i)} x\right)(t)-\left(D_{r}^{(i)} w\right)(t)\right]=0 \quad(i=1,2, \ldots, n-1)
$$

Proof. - Let $L$ be a real number with $|L|>2 A$. Without loss of generality, we assume that $L$ is positive, since the substitution $z=-x$ transforms $(E)$ into an equation of the same form satisfying the assumptions of the theorem. Moreover, we suppose that $L>2 B$, where

$$
B=\max _{0 \leqq i \leqq N} \sup _{t \geqq T_{0}}\left|\left(D_{r}^{(i)} w\right)(t)\right|
$$


for some $T_{0} \geqq t_{0}$. Furthermore, we set

$$
K_{0}=\left\{y_{0}=\left(y_{01}, y_{02}, \ldots, y_{0 m_{0}}\right) \in \boldsymbol{R}^{m_{0}}:\left(\forall j=1,2, \ldots, m_{0}\right) \frac{L}{2} \leqq y_{0 i} \leqq \frac{3 L}{2}\right\}
$$

and, if $N>0$, for $i=1,2, \ldots, N$

$$
K_{i}=\left\{y_{i}=\left(y_{i 1}, y_{i 2}, \ldots, y_{i m_{i}}\right) \in \boldsymbol{R}^{m_{2}}:\left(\forall j=1,2, \ldots, m_{i}\right)\left|y_{i j}\right| \leqq \frac{L}{2}\right\}
$$

Then, because of (ii), we have

$$
M=\max _{\left(y_{0}, y_{1}, \ldots, y_{N}\right) \in K_{0} \times K_{1} \times \ldots \times K_{N}} \Phi\left(y_{0}, y_{1}, \ldots, y_{N}\right)>0 .
$$

Next, by conditions (i) and $\left(C_{2}\right)$, we choose a $T \geqq T_{0}$ so that

$$
g_{i \jmath}(t) \geqq T_{0} \quad \text { for every } t \geqq T \quad\left(j=1,2, \ldots, m_{i} ; i=0,1, \ldots, N\right)
$$

and for any $i, 0 \leqq i \leqq N$,

$$
\left\{\begin{array}{l}
\int_{T}^{\infty}|a(s)| d s \leqq \frac{1}{2 M}(L-2 B), \quad \text { if } i=n-1 \\
\int_{T}^{\infty} \frac{1}{r_{i+1}\left(s_{i+1}\right)} \ldots \int_{s_{n-2}}^{\infty} \frac{1}{r_{n-1}\left(s_{n-1}\right)} \int_{s_{n-1}}^{\infty}|a(s)| d s d s_{n-1} \ldots d s_{i+1} \leqq \frac{1}{2 M}(L-2 B), \\
\text { if } i<n-1 .
\end{array}\right.
$$

Now, let $E$ be the Banach space of all functions on the interval $[T, \infty)$ which have bounded continuous $N$-th $r$-derivative on $[T, \infty)$, endowed with the norm II defined by

$$
|h|=\left\{\begin{array}{l}
\|h\|, \quad \text { if } N=0 \\
\left\|D_{r}^{(N)} h\right\|+\sum_{i=0}^{N-1}\left|\left(D_{r}^{(i)} h\right)(T)\right|, \quad \text { if } N>0 .
\end{array}\right.
$$

Furthermore, let the subset $X$ of $E$ consisting of all functions $x \in E$ with

$$
|x(t)-L| \leqq \frac{L}{2} \quad \text { for every } t \geqq T
$$

and, if $N>0$,

$$
\left[\left(D_{\tau}^{(i)} x\right)(t)\right] \leqq \frac{L}{2} \quad \text { for every } t \geqq T(i=1,2, \ldots, N) .
$$

The set $X$ is obviously nonempty and, as it is easy to see, it is convex and closed. 
Let $x \in X$. By the definition of the set $X$, for every $t \geqq T_{0}$ we obviously have

$$
\left\{\begin{array}{l}
\frac{L}{2} \leqq x^{*}(t) \leqq \frac{3 L}{2} \\
\left|\left(D_{r}^{(i)} x\right)^{*}(t)\right| \leqq \frac{L}{2} \quad(i=1,2, \ldots, N), \text { provided that } N>0,
\end{array}\right.
$$

where for $i=0,1, \ldots, N$

$$
\left(D_{r}^{(i)} x\right)^{*}(t)= \begin{cases}\left(D_{r}^{(i)} x\right)(t), & \text { if } t \geqq T \\ \left(D_{r}^{(i)} x\right)(T), & \text { if } T_{0} \leqq t \leqq T .\end{cases}
$$

So, from (ii) and the definition of the constant $M$ it follows that

$$
0<\Phi\left(x^{*}\left\langle g_{0}(t)\right\rangle,\left(D_{r}^{(1)} x\right)^{*}\left\langle g_{1}(t)\right\rangle, \ldots,\left(D_{r}^{(N)} x\right)^{*}\left\langle g_{N}(t)\right\rangle\right) \leqq M
$$

for all $t \geqq T$. Thus, because of condition $\left(C_{2}\right)$, for any $x \in X$ and every $t \geqq T$

$$
\begin{aligned}
\int_{t}^{\infty} \frac{1}{r_{1}\left(s_{1}\right)} \int_{s_{1}}^{\infty} \frac{1}{r_{2}\left(s_{2}\right)} \ldots \int_{s_{n-2}}^{\infty} \frac{1}{r_{n-1}\left(s_{n-1}\right)} \\
\quad \int_{s_{n-1}}^{\infty}|a(s)| \Phi\left(x^{*}\left\langle g_{0}(s)\right\rangle,\left(D_{r}^{(1)} x\right)^{*}\left\langle g_{1}(s)\right\rangle, \ldots,\left(D_{r}^{(N)} x\right)^{*}\left\langle g_{N}(s)\right\rangle\right) d s d s_{n-1} \ldots d s_{2} d s_{1}<\infty
\end{aligned}
$$

and hence it is easy to see that the formula

$$
\begin{aligned}
& (S x)(t)=L+w(t)+(-1)^{n-1} \int_{t}^{\infty} \frac{1}{r_{1}\left(s_{1}\right)} \int_{s_{1}}^{\infty} \frac{1}{r_{2}\left(s_{2}\right)} \ldots \int_{s_{n-2}}^{\infty} \frac{1}{r_{n-1}\left(s_{n-1}\right)} \\
& \int_{s_{n-1}}^{\infty} a(s) \Phi\left(x^{*}\left\langle g_{0}(s)\right\rangle,\left(D_{r}^{(1)} x\right)^{*}\left\langle g_{1}(s)\right\rangle, \ldots,\left(D_{r}^{(N)} x\right)^{*}\left\langle g_{N}(s)\right\rangle\right) d s d s_{n-1} \ldots d s_{2} d s_{1}, \quad t \geqq T
\end{aligned}
$$

defines a mapping $S: X \rightarrow E$. The mapping $S$ satisfies the assumptions of the Schauder theorem. Namely, it satisfies the following:

a) $s X \subseteq X$

In fact, taking into account (2) and (1), for any $x \in X$ and every $t \geqq T$ we obtain

$$
\begin{aligned}
|(S x)(t)-L| \leqq & |w(t)|+\int_{t}^{\infty} \frac{1}{r_{1}\left(s_{1}\right)} \int_{s_{1}}^{\infty} \frac{1}{r_{2}\left(s_{2}\right)} \ldots \int_{s_{n-2}}^{\infty} \frac{1}{r_{n-1}\left(s_{n-1}\right)} \\
& \int_{s_{n-1}}^{\infty}|a(s)| \Phi\left(x^{*}\left\langle g_{0}(s)\right\rangle,\left(D_{r}^{(1)} x\right)^{*}\left\langle g_{1}(s)\right\rangle, \ldots,\left(D_{r}^{(N)} x\right)^{*}\left\langle g_{N}(s)\right\rangle\right) d s d s_{n-1} \ldots d s_{2} d s_{1} \\
& \leqq \sup _{t \geqq T}|w(t)|+M \int_{T}^{\infty} \frac{1}{r_{1}\left(s_{1}\right)} \int_{s_{1}}^{\infty} \frac{1}{r_{2}\left(s_{2}\right)} \ldots \int_{s_{n-2}}^{\infty} \frac{1}{r_{n-1}\left(s_{n-1}\right)} \\
& \int_{s_{n-1}}^{\infty}|a(s)| d s d s_{n-1} \ldots d s_{2} d s_{1} \leqq B+M \frac{1}{2 M}(L-2 B)=\frac{L}{2}
\end{aligned}
$$


Furthermore, if $N>0$, then, by virtue again of (2) and (1), for $x \in X, t \geqq T$ and any $i, 1 \leqq i \leqq N$, we get

$$
\begin{array}{r}
\left|\left(D_{r}^{(i)}(S x)\right)(t)\right| \leqq\left|\left(D_{r}^{(i)} w\right)(t)\right|+\int_{t}^{\infty}|a(s)| \Phi\left(x^{*}\left\langle g_{0}(s)\right\rangle,\left(D_{r}^{(1)} x\right)^{*}\left\langle g_{1}(s)\right\rangle, \ldots,\left(D_{r}^{(M)} x\right)^{*}\left\langle g_{N}(s)\right\rangle\right) d s \\
\leqq \sup _{t \geqq T}\left|\left(D_{r}^{(i)} w\right)(t)\right|+M \int_{T}^{\infty}|a(s)| d s \leqq B+M \frac{1}{2 M}(L-2 B)=\frac{L}{2},
\end{array}
$$

if $i=n-1$, and

$$
\begin{aligned}
\left|\left(D_{r}^{(i)}(S x)\right)(t)\right| \leqq & \left|\left(D_{r}^{(i)} w\right)(t)\right|+\int_{t}^{\infty} \frac{1}{r_{i+1}\left(s_{i+1}\right)} \ldots \int_{s_{n-2}}^{\infty} \frac{1}{r_{n-1}\left(s_{n-1}\right)} \\
& \int_{s_{n-1}}^{\infty}|a(s)| \Phi\left(s^{*}\left\langle g_{0}(s)\right\rangle,\left(D_{r}^{(1)} x\right)^{*}\left\langle g_{1}(s)\right\rangle, \ldots,\left(D_{r}^{(N)} x\right)^{*}\left\langle g_{N}(s)\right\rangle\right) d s d s_{n-1} \ldots d s_{i+1} \\
& \leqq \sup _{t \geqq T}\left|\left(D_{r}^{(i)} w\right)(t)\right|+M \int_{T}^{\infty} \frac{1}{r_{i+1}\left(s_{i+1}\right)} \ldots \int_{s_{n-2}}^{\infty} \frac{1}{r_{n-1}\left(s_{n-1}\right)} \\
& \int_{s_{n-1}}^{\infty}|a(s)| d s d s_{n-1} \ldots d s_{i+1} \leqq B+M \frac{1}{2 M}(L-2 B)=\frac{L}{2}, \quad \text { if } i<n-1 .
\end{aligned}
$$

B) $\$ X$ is relatively compact.

If $x \in X$ and $f=D_{r}^{(N)}(S x)-D_{r}^{(N)} w$, then, because of the fact that $S X \subseteq X$ and the definition of $X$, for every $t \geqq T$ we have

$$
|f(t)| \leqq\left|\left(D_{r}^{(N)}(S x)\right)(t)\right|+\left|\left(D_{r}^{(N)} w\right)(t)\right| \leqq \frac{3 L}{2}+B .
$$

So, the set

$$
\mathcal{F}=\left\{D_{r}^{(N)}(S x)-D_{r}^{(N)} w: x \in X\right\}
$$

is an uniformly bounded subset of the Banach space $B([T, \infty))$. Furthermore, for any function $f$ in $\mathcal{F}$ and every $t \geqq T$

$$
|\vec{f}(t)| \leqq\left\{\begin{array}{l}
M \int_{i}^{\infty}|a(s)| d s, \quad \text { if } N=n-1 \\
\int_{t}^{\infty} \frac{1}{r_{N+1}\left(s_{N+1}\right)} \ldots \int_{s_{n-2}}^{\infty} \frac{1}{r_{n-1}\left(s_{n-1}\right)} \int_{s_{n-1}}^{\infty}|a(s)| d s d s_{n-1} \ldots d s_{N+1}, \quad \text { if } N<n-1,
\end{array}\right.
$$

where

$$
\hat{f}(t)= \begin{cases}f(t)-L, & \text { if } N=0 \\ f(t), & \text { if } N>0\end{cases}
$$


Indeed, if $f=D_{r}^{(N)}(S x)-D_{r}^{(N)} w, x \in X$, for every $t \geqq T$ we obtain

$$
\begin{aligned}
|\bar{f}(t)|= & \begin{cases}|[(S x)(t)-w(t)]-L|, & \text { if } N=0 \\
\left|\left(D_{r}^{(N)}(S x)\right)(t)-\left(D_{r}^{(N)} w\right)(t)\right|, & \text { if } N>0\end{cases} \\
& \leqq \begin{cases}\int_{t}^{\infty}|a(s)| \Phi\left(x^{*}\left\langle g_{0}(s)\right\rangle,\left(D_{r}^{(1)} x\right)^{*}\left\langle g_{1}(s)\right\rangle, \ldots,\left(D_{r}^{(N)} x\right)^{*}\left\langle g_{N}(s)\right\rangle\right) d s, & \text { if } N=n-1 \\
\int_{t}^{\infty} \frac{1}{r_{N+1}\left(s_{N+1}\right)} \ldots \int_{s_{n-2}}^{\infty} \frac{1}{r_{n-1}\left(s_{n-1}\right)} \int_{s_{n-1}}^{\infty}|a(s)| \Phi\left(x^{*}\left\langle g_{0}(s)\right\rangle,\left(D_{r}^{(1)} x\right)^{*}\left\langle g_{1}(s)\right\rangle, \ldots\right. \\
\left.\ldots,\left(D_{r}^{(N)} x\right)^{*}\left\langle g_{N}(s)\right\rangle\right) d s d s_{n-1} \ldots d s_{N+1}, & \text { if } N<n-1\end{cases}
\end{aligned}
$$

and hence, by virtue of (2), (3) follows immediately. From (3) and condition $\left(C_{2}\right)$ it follows that the set $\mathfrak{F}$ is equiconvergent at $\infty$. Now, by using again (2), for any function $f \in \mathcal{F}^{*}$ and every $t_{1}, t_{2}$ with $T \leqq t_{1} \leqq t_{2}$ we can easily obtain

$$
\begin{aligned}
\left|f\left(t_{2}\right)-f\left(t_{1}\right)\right| & \leqq \\
& \leqq
\end{aligned} \begin{aligned}
& M \int_{t_{1}}^{t_{3}}|a(s)| d s, \quad \text { if } N=n-1 \\
& M \int_{t_{1}}^{t_{2}} \frac{1}{r_{n-1}\left(s_{n-1}\right)} \int_{s_{n-1}}^{\infty}|a(s)| d s d s_{n-1}, \quad \text { if } N=n-2 \\
& M \int_{t_{1}}^{t_{2}} \frac{1}{r_{N+1}\left(s_{N+1}\right)} \int_{s_{N+1}}^{\infty} \frac{1}{r_{N+2}\left(s_{N+2}\right)} \ldots \int_{s_{n-2}}^{\infty} \frac{1}{r_{n-1}\left(s_{n-1}\right)} \int_{s_{n-1}}^{\infty}|a(s)| d s d s_{n-1} \ldots d s_{N+2} d s_{N+1},
\end{aligned}
$$

By the last inequality, it is easy to verify that $\mathscr{F}$ is equicontinuous. Finally, by the compactness criterion, we conclude that the set $\mathcal{F}$ is relatively compact in $B([T, \infty))$. This, because of the definition of the norm I In $E$ and the boundedness of the function $D_{r}^{(N)} w$, implies the relative compactness of the set $S X$.

\section{$\gamma)$ The mapping $S$ is continuous.}

Let $x \in X$ and $\left(x_{v}\right)$ be an arbitrary sequence in $X$ with

$$
\mid 1-\lim x_{y}=x
$$

Then it is easy to verify that for every $t \geqq T_{0}$

$$
\lim _{v}\left(D_{r}^{(i)} x_{v}\right)^{*}(t)=\left(D_{r}^{(i)} x\right)^{*}(t) \quad(i=0,1, \ldots, N) .
$$


On the other hand, by (2), for all $\nu$ and every $t \geqq T$ we have

$$
\left|a(t) \Phi\left(x_{v}^{*}\left\langle g_{0}(t)\right\rangle,\left(D_{r}^{(1)} x_{\nu}\right)^{*}\left\langle g_{1}(t)\right\rangle, \ldots,\left(D_{r}^{(N)} x_{v}\right)^{*}\left\langle g_{N}(t)\right\rangle\right)\right| \leqq M|a(t)| .
$$

Thus, because of condition $\left(C_{2}\right)$, we can apply the Lebesgue dominated convergence theorem to obtain

$$
\begin{aligned}
\lim _{v} \int_{i}^{\infty} \frac{1}{r_{1}\left(s_{1}\right)} \int_{s_{1}}^{\infty} \frac{1}{r_{2}\left(s_{2}\right)} \ldots \int_{s_{n-2}}^{\infty} \frac{1}{r_{n-1}\left(s_{n-1}\right)} \\
\quad \int_{s_{n-1}}^{\infty} a(s) \Phi\left(x_{\nu}^{*}\left\langle g_{0}(s)\right\rangle,\left(D_{r}^{(1)} x_{\nu}\right)^{*}\left\langle g_{1}(s)\right\rangle, \ldots,\left(D_{r}^{(N)} x_{\nu}\right)^{*}\left\langle g_{N}(s)\right\rangle\right) d s d s_{n-1} \ldots d s_{2} d s_{1} \\
\quad=\int_{i}^{\infty} \frac{1}{r_{1}\left(s_{1}\right)} \int_{s_{1}}^{\infty} \frac{1}{r_{2}\left(s_{2}\right)} \ldots \int_{s_{n-2}}^{\infty} \frac{1}{r_{n-1}\left(s_{n-1}\right)} \\
\\
\quad \int_{s_{n-1}}^{\infty} a(s) \Phi\left(x^{*}\left\langle g_{0}(s)\right\rangle,\left(D_{r}^{(1)} x\right)^{*}\left\langle g_{1}(s)\right\rangle, \ldots,\left(D_{r}^{(N)} x\right)^{*}\left\langle g_{N}(s)\right\rangle\right) d s d s_{n-1} \ldots d s_{2}^{\prime} d s_{1}
\end{aligned}
$$

for all $t \geqq T$. So, for every $t \geqq T$ we have the pointwise convergence

$$
\lim _{\nu}\left(S x_{v}\right)(t)=(S x)(t)
$$

It remains to prove that

$$
11-\lim s x_{\nu}=S x
$$

To this end, we consider any subsequence $\left(u_{\mu}\right)$ of $\left(S x_{v}\right)$. Becanse of the relative compactness of $S X$, there exist a subsequence $\left(v_{\lambda}\right)$ of $\left(u_{\mu}\right)$ and a $y \in E$ so that

$$
||-\lim v_{\lambda}=y \text {. }
$$

Since I|-convergence implies the pointwise one to the same limit function, me must always have $y=S x$, which proves $(4)$.

Finally, by the Schauder theorem, there exists an $x \in X$ with $x=S x$. Then, in view of condition $\left(C_{1}\right)$, we have

$$
\begin{aligned}
\left(D_{r}^{(n)} x\right)(t) & =\left(D_{r}^{(n)} w\right)(t)-a(t) \Phi\left(x^{*}\left\langle g_{0}(t)\right\rangle,\left(D_{r}^{(1)} x\right)^{*}\left\langle g_{1}(t)\right\rangle, \ldots,\left(D_{r}^{(N)} x\right)^{*}\left\langle g_{N}(t)\right\rangle\right) \\
& =b(t)-a(t) \Phi\left(x^{*}\left\langle g_{0}(t)\right\rangle,\left(D_{r}^{(1)} x\right)^{*}\left\langle g_{1}(t)\right\rangle, \ldots,\left(D_{r}^{(N)} x\right)^{*}\left\langle g_{N}(t)\right\rangle\right)
\end{aligned}
$$

for every $t \geqq T$. Thus, the fixed point $x$ of the mapping $S$ is a solution on $[T, \infty)$ 
of the equation $(E)$. Moreover, this solution is the required one, since

$$
\begin{array}{r}
|[x(t)-w(t)]-L| \leqq \int_{i}^{\infty} \frac{1}{r_{1}\left(s_{1}\right)} \int_{s_{1}}^{\infty} \frac{1}{r_{2}\left(s_{2}\right)} \ldots \int_{s_{n-2}}^{\infty} \frac{1}{r_{n-1}\left(s_{n-1}\right)} \int_{s_{n-1}}^{\infty}|a(s)| \Phi\left(x^{*}\left\langle g_{0}(s)\right\rangle,\left(D_{r}^{(1)} x\right)^{*}\left\langle g_{1}(s)\right\rangle, \ldots\right. \\
\left.\ldots,\left(D_{r}^{(N)} x\right)^{*}\left\langle g_{N}(s)\right\rangle\right) d s d s_{n-1} \ldots d s_{2} d s_{1} \rightarrow 0 \text { as } t \rightarrow \infty
\end{array}
$$

and for any $i, 1 \leqq i \leqq n-1$,

$$
\begin{aligned}
& \left|\left(D_{r}^{(i)} x\right)(t)-\left(D_{r}^{(2)} w\right)(t)\right| \leqq \\
& \leqq\left\{\begin{array}{l}
\int_{i}^{\infty}|a(s)| \Phi\left(x^{*}\left\langle g_{0}(s)\right\rangle,\left(D_{r}^{(1)} x\right)^{*}\left\langle g_{1}(s)\right\rangle, \ldots,\left(D_{r}^{(s)} x\right)^{*}\left\langle g_{N}(s)\right\rangle\right) d s, \quad \text { if } i=n-1 \\
\int_{i}^{\infty} \frac{1}{r_{i+1}\left(s_{i+1}\right)} \ldots \int_{s_{n-2}}^{\infty} \frac{1}{r_{n-1}\left(s_{n-1}\right)} \int_{s_{n-1}}^{\infty}|a(s)| \Phi\left(x^{*}\left\langle g_{0}(s)\right\rangle,\left(D_{r}^{(1)} x\right)^{*}\left\langle g_{1}(s)\right\rangle, \ldots\right. \\
\left.\ldots,\left(D_{r}^{(N)} x\right)^{*}\left\langle g_{N}(s)\right\rangle\right) d s d s_{n-1} \ldots d s_{i+1}, \quad \text { if } i<n-1
\end{array}\right.
\end{aligned}
$$

$\rightarrow 0$ as $t \rightarrow \infty$.

Corollary I below follows immediately from Theorem 1 .

COROLLARY I. - Consider the differential equation (E) subject to the conditions (i), (ii), $\left(C_{2}\right)$ and:

$\left(C_{1}^{\prime}\right)$ There exists an $n$-times continuously $r$-differentiable function $w$ on $\left[t_{0}, \infty\right)$ with $D_{r}^{(n)} w=b$ and such that

$$
\lim _{t \rightarrow \infty}\left(D_{r}^{(i)} w\right)(t)=0 \quad(i=0,1, \ldots, N) .
$$

Then for every real number $L$ with $L \neq 0$ there exists a (bounded nonoscillatory) solution $x$ of the equation $(E)$ with

$$
\lim _{t \rightarrow \infty} \infty(t)=L
$$

and, if $N>0$,

$$
\lim _{t \rightarrow \infty}\left(D_{r}^{(i)} x\right)(t)=0 \quad(i=1,2, \ldots, N) .
$$

In particular, for $b=0$ and $v=0$, condition $\left(C_{1}\right)$ is satisfied. Thus, from Theorem 1 we immediately obtain the following corollary concerning the differential equation

$\left(E_{0}\right) \quad\left(D_{r}^{(n)} x\right)(t)+a(t) \Phi\left(x\left\langle g_{0}(t)\right\rangle,\left(D_{r}^{(1)} x\right)\left\langle g_{1}(t)\right\rangle, \ldots,\left(D_{r}^{(N)} x\right)\left\langle g_{N}(t)\right\rangle\right)=0$.

COROLLARY II. - Consider the differential equation $\left(E_{0}\right)$ subject to the conditions (i), (ii) and $\left(C_{2}\right)$. Then for every real number $L$ with $L \neq 0$ there exists a (bounded nooscilla-

3 - Annali di Matematica 
tory) solution $x$ of the equation $\left(E_{0}\right)$ with

$$
\lim _{t \rightarrow \infty} x(t)=L \quad \text { and } \quad \lim _{t \rightarrow \infty}\left(D_{r}^{(i)} x\right)(t)=0 \quad(i=1,2, \ldots, n-1) .
$$

In our previous results (Theorem 1 and its corollaries) no restriction on the sign of the function $a$ is assumed. Moreover, the only assumption on the functions $r_{\imath}$ $(i=1,2, \ldots, n-1)$ is that they are positive and continuous on the interval $\left[t_{0}, \infty\right)$. Now, in order to obtain sufficient conditions for the oscillatory and asymptotic behavior of the bounded solutions of the differential equation $\left(E_{0}\right)$ we shall restrict ourselves in the case where the function $a$ is of constant sign on $\left[t_{0}, \infty\right)$ and the condition $(R)$ below is satisfied:

(R) For every $i=1,2, \ldots, n-1$

$$
\int_{r_{i}(t)}^{\infty}=\infty
$$

We need the following lemma due to PHrLos [4, Lemmas 1 and 2].

LEMMA. - Suppose that condition $(R)$ is satisfied. Let $\delta= \pm 1$ and $h$ be a positive bounded and $n$-times r-differentiable function on the interval $[T, \infty), T \geqq t_{0}$, with

$$
\delta\left(D_{r}^{(n)} h\right)(t) \leqq 0 \quad \text { for every } t \geqq T .
$$

Then

$$
\begin{gathered}
\delta(-1)^{n+1+i}\left(D_{r}^{(i)} h\right)(t) \geqq 0 \quad \text { for every } t \geqq T \quad(i=1,2, \ldots, n-1), \\
\lim _{t \rightarrow \infty}\left(D_{r}^{(i)} h\right)(t)=0 \quad(i=1,2, \ldots, n-1)
\end{gathered}
$$

and, provided that $D_{r}^{(n)} h$ is continuous on $[T, \infty)$,

$$
\int_{T}^{\infty} \frac{1}{r_{1}\left(s_{1}\right)} \int_{s_{1}}^{\infty} \frac{1}{r_{2}\left(s_{2}\right)} \ldots \int_{s_{n-2}}^{\infty} \frac{1}{r_{n-1}\left(s_{n-1}\right)} \int_{s_{n-1}}^{\infty}\left|\left(D_{r}^{(n)} h\right)(s)\right| d s d s_{n-1} \ldots d s_{2} d s_{1}<\infty .
$$

THEOREM 2. - Consider the differential equation $\left(E_{0}\right)$ subject to the conditions (i), (ii), $(R)$ and:

$\left(C_{3}\right)$ There exists an integer $k$ with $0 \leqq k \leqq n-1$ and such that

$$
\left\{\begin{array}{l}
\int^{\infty}|a(t)| d t=\infty, \quad \text { if } k=n-1 \\
\int^{\infty} \frac{1}{r_{k+1}\left(s_{k+1}\right)} \ldots \int_{s_{n-2}}^{\infty} \frac{1}{r_{n-1}\left(s_{n-1}\right)} \int_{s_{n-1}}^{\infty}|a(s)| d s d s_{n-1} \ldots d s_{k+1}=\infty, \quad \text { if } k<n-1 .
\end{array}\right.
$$


If the function $a$ is nonnegative (respectively, nonpositive) on the interval $\left[t_{0}, \infty\right)$, then every bounded solution $x$ of the equation $\left(E_{0}\right)$ for $n$ even (resp. odd) is oscillatory while for $n$ odd (resp. even) is either oscillatory or such that

$$
\lim _{t \rightarrow \infty}\left(D_{r}^{(i)} x\right)(t)=0 \quad \text { monotonically }(i=0,1, \ldots, n-1)
$$

Proof, - Let $x$ be a bounded nonoscillatory solution on an interval $\left[T_{0}, \infty\right), T_{0} \geqq t_{0}$, of the equation $\left(E_{0}\right)$. Without loss of generality, we suppose that $x(t) \neq 0$ for every $t \geqq T_{0}$. Furthermore, we assume that $x$ is positive, since the substitution $z=-x$ transforms $\left(E_{0}\right)$ into an equation of the same form satisfying the assumptions of the theorem.

By (i), we choose a $T \geqq T_{0}$ so that

$$
g_{i j}(t) \geqq T_{0} \quad \text { for every } t \geqq T \quad\left(j=1,2, \ldots, m_{i} ; i=0,1, \ldots, N\right) .
$$

Then, in view of (ii), from equation $\left(E_{0}\right)$ we obtain

$$
-\delta\left(D_{r}^{(n)} x\right)(t)=|a(t)| \Phi\left(x\left\langle g_{0}(t)\right\rangle,\left(D_{r}^{(1)} x\right)\left\langle g_{1}(t)\right\rangle, \ldots,\left(D_{r}^{(N)} x\right)\left\langle g_{N}(t)\right\rangle\right) \geqq 0
$$

for all $t \geqq T$, where

$$
\delta=\left\{\begin{aligned}
+1, & \text { if } a \geqq 0 \\
-1, & \text { if } a \leqq 0
\end{aligned}\right.
$$

Namely,

$$
\delta\left(D_{r}^{(n)} x\right)(t) \leqq \mathbf{0} \quad \text { for every } t \geqq T
$$

Thus, by the lemma, we have

$$
\begin{gathered}
\delta(-1)^{n+1+i}\left(D_{r}^{(i)} x\right)(t) \geqq 0 \quad \text { for every } t \geqq T \quad(i=1,2, \ldots, n-1), \\
\lim _{t \rightarrow \infty}\left(D_{r}^{(i)} x\right)(t)=0 \quad(i=1,2, \ldots, n-1)
\end{gathered}
$$

and

$$
\int \frac{1}{r_{1}\left(s_{1}\right)} \int_{s_{1}}^{\infty} \frac{1}{r_{2}\left(s_{2}\right)} \ldots \int_{s_{n-2}}^{\infty} \frac{1}{r_{n-1}\left(s_{n-1}\right)} \int_{s_{n-1}}^{\infty}\left|\left(D_{r}^{(n)} x\right)(s)\right| d s d s_{n-1} \ldots d s_{2} d s_{1}<\infty
$$

The functions $D_{r}^{(i)} i(i=0,1, \ldots, N)$ are obviously bounded.

Now, it holds

$$
\lim _{t \rightarrow \infty} x(t)=0
$$

Indeed, in the opposite case for some positive constant $K$

$$
o(t) \geqq K \quad \text { for every } t \geqq T_{\mathbf{0}} \text {. }
$$


So, because of (ii), the boundedness of the functions $D_{r}^{(i)} x(i=0,1, \ldots, N)$ and the continuity of the function $\Phi$, there exists a positive constant $e$ such that for every $t \geqq T$

$$
\Phi\left(x\left\langle g_{0}(t)\right\rangle,\left(D_{r}^{(1)} x\right)\left\langle g_{1}(t)\right\rangle, \ldots,\left(D_{r}^{(N)} x\right)\left\langle g_{N}(t)\right\rangle\right) \geqq 0
$$

Therefore,

$$
\left|\left(D_{r}^{(n)} x\right)(t)\right| \geqq o|a(t)| \quad \text { for all } t \geqq T
$$

and hence (7) gives

$$
\int_{T}^{\infty} \frac{1}{r_{1}\left(s_{1}\right)} \int_{s_{1}}^{\infty} \frac{1}{r_{2}\left(s_{2}\right)} \ldots \int_{s_{n-2}}^{\infty} \frac{1}{r_{n-1}\left(s_{n-1}\right)} \int_{s_{n-1}}^{\infty}|a(s)| d s d s_{n-1} \ldots d s_{2} d s_{1}<\infty
$$

which contradicts $\left(C_{3}\right)$.

Finally, by (5) and (8), we conclude that $\delta(-1)^{n}=-1$, namely that $n$ is odd (resp. even) if $\delta=+1$ (resp. $\delta=-1$ ). Thus, (5), (6) and (8) prove the theorem.

CoRoLLARY III. - Consider the differential equation $\left(E_{0}\right)$ subject to the conditions (i), (ii) and $(R)$. If the function a is of constant sign on $\left[t_{0}, \infty\right)$, then the condition $\left(O_{2}\right)$ is a necessary and sufficient condition in order that the equation $\left(E_{0}\right)$ have at least one (bounded nonosoillatory) solution $x$ so that the $\lim _{t \rightarrow \infty} x(t)$ exists in $\boldsymbol{R}-\{0\}$.

Proof. - The necessity of $\left(C_{2}\right)$ follows immediately from Theorem 3 , while its sufficiency is contained in Corollary II.

\section{3. - Applications.}

We shall now clarify the importance of the results of Section 2 by applying them in the particular ease where for some integer $m, 1 \leqq m \leqq n-1$, we have

$$
r_{i}=1 \quad \text { for } i \neq n-m \text { and } \quad r_{n-m}=r .
$$

In this case the equation $(E)$ takes the form

$$
\left[r(t) x^{(n-m)}(t)\right]^{(m)}+a(t) \Phi\left(x\left\langle g_{0}(t)\right\rangle, x^{\prime}\left\langle g_{1}(t)\right\rangle, \ldots, x^{(N)}\left\langle g_{N}(t)\right\rangle\right)=b(t),
$$

if $N<n-m$, and the form

$(\hat{E}) \quad\left[r(t) x^{(n-m)}(t)\right]^{(n)}+a(t) \Phi\left(x\left\langle g_{0}(t)\right\rangle, x^{\prime}\left\langle g_{1}(t)\right\rangle, \ldots, x^{(n-m-1)}\left\langle g_{n-m-1}(t)\right\rangle\right.$,

$$
\left.\left[r x^{(n-m)}\right]\left\langle g_{n-m}(t)\right\rangle,\left[r x^{(n-m)}\right]^{\prime}\left\langle g_{n^{-m+1}}(t)\right\rangle, \ldots,\left[r x^{(n-m)}\right]^{(N-(n-m))}\left\langle g_{N}(t)\right\rangle\right)=b(t),
$$


if $N \geqq n-m$. Also, the condition $(R)$ becomes:

$$
\int^{\infty} \frac{d t}{r(t)}=\infty
$$

Let $\left(\hat{E}_{0}\right)$ denote the differential equation $(\hat{E})$ with $b=0$. Corollaries $1,2,3,4$ and 5 below are now and follow from Theorem 1, Corollary I, Corollary II, Theorem 2 and Corollary III respectively.

CoRollaRY 1. - Consider the differential equation $(\hat{E})$ subject to the conditions (i), (ii) and:

$\left(\hat{C}_{1}\right)$ There exists a function $w \in C^{n-m}\left(\left[t_{0}, \infty\right)\right)$ with $r w^{(n-m)} \in C^{m}\left(\left[t_{0}, \infty\right)\right)$ and $\left[r w^{(n-m)}\right]^{(m)}=b$ and such that $\hat{A}<\infty$, where

$$
\hat{A}=\left\{\begin{array}{l}
\max _{0 \leqq i \leqq N} \limsup _{t \rightarrow \infty}\left|w^{(i)}(t)\right|, \quad \text { if } N<n-m \\
\max \left\{\max _{0 \leq i \leq n-m-1} \limsup _{t \rightarrow \infty}\left|w^{(i)}(t)\right|, \max _{0 \leqq j \leqq N-(n-m)} \limsup _{t \rightarrow \infty}\left|\left[r(t) w^{(n-m)}(t)\right]^{(j)}\right|\right\}, \\
\text { if } N \geqq n-m .
\end{array}\right.
$$

$\left(\hat{C_{2}}\right)$

$$
\int^{\infty} \frac{t^{n-1-m}}{r(t)} \int_{i}^{\infty}(s-t)^{m-1}|a(s)| d s d t<\infty
$$

Then for every real number $L$ with $|L|>2 \hat{A}$ there exists a (bounded nonoscillatory) solution $x$ of the equation $(\hat{E})$ with

$$
\lim _{t \rightarrow \infty}[x(t)-w(t)]=L
$$

and

$$
\left\{\begin{array}{l}
\lim _{t \rightarrow \infty}\left[x^{(i)}(t)-w^{(2)}(t)\right]=0 \quad(i=1,2, \ldots, n-m-1), \text { if } m<n-1 \\
\lim _{t \rightarrow \infty}\left(\left[r(t) x^{(n-m)}(t)\right]^{(j)}-\left[r(t) w^{(n-m)}(t)\right]^{(\jmath)}\right)=0 \quad(j=0,1, \ldots, m-1) .
\end{array}\right.
$$

Coroutary 2. - Consider the differential equation $(\hat{E})$ subject to the conditions (i), (ii), $\left(\hat{C}_{2}\right)$ and:

$\left(\hat{C}_{1}^{\prime}\right)$ There exists a function $w \in C^{n-m}\left(\left[t_{0}, \infty\right)\right)$ with $r w^{(n-m)} \in C^{m}\left(\left[t_{0}, \infty\right)\right)$ and $\left[r w^{(n-m)}\right]^{(m)}=b$ and such that:

$$
\lim _{t \rightarrow \infty} w^{(2)}(t)=0 \quad(i=0,1, \ldots, N)
$$


if $N<n-m$, and

$$
\left\{\begin{array}{l}
\lim _{t \rightarrow \infty} w^{(2)}(t)=0 \quad(i=0,1, \ldots, n-m-1) \\
\lim _{t \rightarrow \infty}\left[r(t) w^{(n-m)}(t)\right]^{(\jmath)}=0 \quad(j=0,1, \ldots, N-(n-m)),
\end{array}\right.
$$

if $N \geqq n-m$.

Then for every real number $L$ with $L \neq 0$ there exists a (bounded nonoscillatory) solution $x$ of the equation $(\hat{E})$ with

$$
\lim _{t \rightarrow \infty} x(t)=L
$$

and, provided that $N>0$,

$$
\lim _{t \rightarrow \infty} \mathfrak{x}^{(i)}(t)=0 \quad(i=1,2, \ldots, N),
$$

if $N<n-m$, and

$$
\left\{\begin{array}{l}
\lim _{t \rightarrow \infty} x^{(i)}(t)=0 \quad(i=1,2, \ldots, n-m-1), \text { when } m<n-1 \\
\lim _{t \rightarrow \infty}\left[r(t) x^{(n-m)}(t)\right]^{(j)}=0 \quad(j=0,1, \ldots, N-(n-m))
\end{array}\right.
$$

if $N \geqq n-m$.

CorolLary 3. - Consider the differential equation $\left(\hat{E}_{0}\right)$ subject to the conditions (i), (ii) and $\left(\hat{C}_{2}\right)$. Then for every real number $L$ with $L \neq 0$ there exists a (bounded nonoscillatory) solution $x$ of the equation $\left(\hat{E}_{0}\right)$ with

and

$$
\lim _{t \rightarrow \infty} x(t)=L
$$

$$
\left\{\begin{array}{l}
\lim _{t \rightarrow \infty} x^{(i)}(t)=0 \quad(i=1,2, \ldots, n-m-1), \text { if } m<n-1 \\
\lim _{t \rightarrow \infty}\left[r(t) x^{(n-m)}(t)\right]^{(j)}=0 \quad(j=0,1, \ldots, m-1)
\end{array}\right.
$$

CoRoLlaRT 4. - Consider the differential equation $\left(\hat{E}_{0}\right)$ subject to the conditions (i), (ii), $(\hat{R})$ and:

$\left(\hat{C}_{3}\right)$ Either

$$
\int^{\infty} t^{m-1}|a(t)| d t=\infty
$$

or

$$
\int^{\infty} \frac{t^{n-1-m}}{r(t)} \int_{i}^{\infty}(s-t)^{m-1}|a(s)| d s d t=\infty
$$


If the function a is nonnegative (resp. nonpositive) on $\left[t_{0}, \infty\right)$, then every bounded solution $x$ of the equation $\left(\hat{E}_{0}\right)$ for $n$ even (resp. odd) is oscillatory, while for $n$ odd (resp. even) is either oscitlatory or such that

$$
\left\{\begin{array}{l}
\lim _{t \rightarrow \infty} x^{(i)}(t)=0 \quad \text { monotonically }(i=0,1, \ldots, n-m-1) \\
\lim _{t \rightarrow \infty}\left[r(t) x^{(n-m)}(t)\right]^{(\jmath)}=0 \quad \text { monotonically }(j=0,1, \ldots, m-1) .
\end{array}\right.
$$

CoROLLART 5. - Consider the differential equation $\left(\hat{E}_{0}\right)$ subject to the conditions (i), (ii) and $(\hat{R})$. If the function $a$ is of constant sign on $\left[t_{0}, \infty\right)$, then the condition $\left(\hat{C}_{2}\right)$ is a necessary and sufficient condition in order that the equation $\left(\hat{E}_{0}\right)$ have at least one (bounded nonoscillatory) solution $x$ so that the $\lim _{t \rightarrow \infty} x(t)$ exists in $\boldsymbol{R}-\{0\}$.

It is easy to see that in the considered case the conditions $\left(C_{1}\right)$ and $\left(C_{1}^{\prime}\right)$ become $\left(\hat{C}_{1}\right)$ and $\left(\hat{C}_{1}^{\prime}\right)$ respectively. Furthermore, for any nonnegative integer $\mu$ and a nonnegative continuous function $p$ on $[u, \infty)$ we have the formula

$$
\int_{u}^{\infty} \int_{v}^{\infty}(s-v)^{\mu} p(s) d s=\int_{u}^{\infty} \frac{(s-u)^{\mu+1}}{\mu+1} p(s) d s .
$$

By this formula, it is a matter of elementary calculus (cf. [4]) to verify that the conditions $\left(C_{2}\right)$ and $\left(C_{3}\right)$ follow from $\left(\hat{C}_{2}\right)$ and $\left(\hat{C}_{3}\right)$ respectively.

Before closing this paper, let us consider the usual case where $r_{1}=r_{2}=\ldots=$ $=r_{n-1}=1$. In this case the equation $(E)$ takes the form

$$
x^{(n)}(t)+a(t) \Phi\left(x\left\langle g_{0}(t)\right\rangle, x^{\prime}\left\langle g_{1}(t)\right\rangle, \ldots, x^{(N)}\left\langle g_{N}(t)\right\rangle\right)=b(t),
$$

the condition $(R)$ holds by itself and (cf. [4]) the conditions $\left(C_{2}\right)$ and $\left(C_{3}\right)$ become

$$
\int^{\infty} t^{n-1}|a(t)| d t<\infty
$$

and

$$
\int^{\infty} t^{n-1}|a(t)| d t=\infty
$$

respectively. Thus, the results of Section 2 can be easily formulated for the differential equation $(\widetilde{E})$ so that to generalize some results of the book of STAIKos [10].

Acknowledgment. - The author would like to thank Professor V. A. STaIKos for his helpful suggestions concerning this paper. 


\section{REFERENCES}

[1] W. A. Coppex, Disconjugacy, Lecture Notes in Mathematics 220, Springer-Verlag, 1971.

[2] T. Kusano - H. OnOsE, Asymptotio behavior of nonoscillatory solutions of functional differential equations of arbitrary order, J. London Math. Soc., 14 (1976), pp. 106-112.

[3] T. Kusano - H. Onose, Nonoscillation theorems for differential equations with deviating argument, Pacific J. Math., 63 (1976), pp. 185-192.

[4] Cr. G. PHILos, Oscillatory and asymptotic behavior of the bounded solutions of differential equations with deviating arguments, Hiroshima Math. J., 8 (1978), pp. 31-48.

[5] Cr. G. PhrLos, An oscillatory and asymptotic classification of the solutions of differential equations with deviating arguments, Atti Accad. Naz. Lincei, Rend. Cl. Sci. Fis. Mat. Natur., 63 (1977), pp. 195.203.

[6] Сн. G. PHuLos, Oscillatory and asymptotic behavior of all solutions of differential equations with deviating arguments, Proc. Roy. Soc. Edinburgh Sect. A, in press.

[7] Ch. G. Phros - V. A. Stamos, Non-slow oscillations with damping, University of Ioannina, Technical Report no. 92, March 1977.

[8] Ch. G. Philos - V. A. Staikos, Quick oscillations with damping, Math. Nachr., to appear.

[9] J. SCHAUDER, Der Fixpunltsatz in Funltionalläumen, Studia Math., 2 (1930), pp. 171-180.

[10] V. A. SтAIKos, Differential equations with deviating arguments. Oscillation theory (unpublished manuscripts).

[11] V. A. STAIKos - CH. G. PHILOS, On the asymptotic behavior of nonoscillatory solutions of differential equations with deviating arguments, Hiroshima Math. J., 7 (1977), pp. 9-31.

[12] V. A. Statkos - CH. G. Philos, Asymptotie properties of nonoscillatory solutions of differential equations with deviating argument, Pacific J. Math., 70 (1977), pp. 221-242.

[13] V. A. Stramos - CH. G. PHILos, Nonoscillatory phenomena and damped oscillations, Non linear Analysis, Theory, Methods and Applications, 2 (1977), pp. 197-210.

[14] W. F. TRENCH, Oscillation properties of perturbed disconjugate equations, Proc. Amer. Math. Soc., 52 (1975), pp. 147-155. 